\section{Individual Rights,}

Collective Good and the

\section{Duty of Care}

The ongoing debate over whether or not to be vaccinated has gone beyond the typical ethical dilemma between individual rights and collective good. It now centres on the duty of care. Cost-benefit analysis tends to show that the scales tip in favour of vaccination when considered from the perspective of society. It tips in favour of no vaccination when seen from the perspective of the individual - but only when the majority of people in the community are immunized. The more people who choose no vaccination, the less overall benefit there is to both individuals and society, until such point that herd immunity is lost and outbreaks occur. This scenario of diminishing returns provides the motivation to re-examine this issue in terms of reciprocal duty of care.

Duty of care is equally applicable to individuals and the collective. For example, every health care worker has a duty to not transmit infectious diseases to patients. The National Advisory Committee on Immunization recently stated unequivocally that health care workers who do not get annual influenza vaccine are failing in their duty of care. However, every vaccine carries risk. What is the collective duty towards those who assume this risk?

The duty of care debate is highlighted in this issue, with a focus on both health care workers and children. Gemmill makes an eloquent argument that all health care workers have a duty to get their annual influenza vaccine. Gallant et al. promote a multiple intervention approach to optimize vaccination rates. Bigham et al. note that the main reason for refusal of hepatitis $B$ vaccine in children in British Columbia is parental concern about side effects. Wilson et al. propose ways to address concerns regarding paediatric vaccines that may have relevance for vaccines in general: increase investment in adverse event reporting systems, consider a no-fault compensation program, and develop strategies to decrease risk and improve communication.

The inquiry on what constitutes an appropriate duty of care is evolving and vaccination is a useful lens through which to consider it. The willingness of individuals to assume risk for the collective good is linked to perceived protection and reciprocal care. Although the duty of care towards vaccine recipients is well accepted, there may be ways to optimize the collective management of vaccine risks in order to maximize the benefit for all.

Patricia Huston

Scientific Editor
LE MOT DE LA RÉDACTION

\section{Droits individuels, bien collectif et devoir de diligence}

Le débat sur la nécessité de se faire vacciner ne porte plus seulement sur le vieux dilemme moral entre les droits individuels et le bien collectif : il s'articule maintenant autour du devoir de diligence. Une analyse coûts-avantages a tendance à montrer que la balance penche du côté de la vaccination lorsqu'on adopte une perspective sociétale, et du côté de la non-vaccination lorsqu'on adopte une perspective individuelle - mais uniquement quand la majorité des membres de la collectivité sont vaccinés. Plus les gens refusent de se faire vacciner, moins ce choix représente un avantage global pour les individus et pour la société. Lorsqu'un certain seuil est dépassé, l'immunité de groupe se perd, et des épidémies surviennent. Ce scénario des rendements décroissants devrait nous motiver à revoir la question sous l'angle de notre devoir de diligence réciproque.

Le devoir de diligence vaut à la fois pour les individus et pour la collectivité. Par exemple, chaque travailleuse et chaque travailleur de la santé a le devoir de ne pas transmettre de maladies infectieuses à ses patients. Le Comité consultatif national de l'immunisation l'a d'ailleurs énoncé récemment en termes catégoriques : les travailleurs de la santé qui ne reçoivent pas le vaccin antigrippal chaque année manquent à leur devoir de diligence. Mais chaque vaccin comporte des risques. Quelle est notre devoir collectif envers les personnes qui acceptent de prendre ces risques?

Il est beaucoup question du devoir de diligence dans ce numéro, et le débat porte à la fois sur les travailleurs de la santé et sur les enfants. Gemmill défend avec éloquence le point de vue selon lequel tous les travailleurs de la santé devraient recevoir annuellement le vaccin antigrippal. Dans l'article de Gallant et coll., on avance le principe des interventions multiples pour optimiser les taux de vaccination. Dans celui de Bigham et coll., on fait valoir que si les parents de la Colombie-Britannique refusent de faire vacciner leurs enfants contre l'hépatite B, c'est d'abord parce qu'ils craignent les effets secondaires du vaccin. Et Wilson et ses collègues proposent des moyens d'apaiser les craintes suscitées par les vaccins pédiatriques - et peut-être par les vaccins en général : investir davantage dans les systèmes de notification des manifestations indésirables, envisager un programme d'indemnisation sans égard à la responsabilité, et élaborer des stratégies pour réduire les risques et améliorer la communication.

Nos interrogations sur ce qui constitue un devoir de diligence adéquat ne cessent d'évoluer, et le débat sur la vaccination permet de clarifier les enjeux. La volonté individuelle d'accepter de prendre des risques pour le bien collectif est liée à la perception de notre niveau de protection et de notre devoir de diligence réciproque. Le devoir de diligence envers les personnes vaccinées est un principe bien accepté, mais il pourrait exister des moyens d'optimiser notre gestion collective des risques des vaccins pour en maximiser les avantages pour tous.

La rédactrice-réviseure scientifique, Patricia Huston 